Erratum

\title{
Intra- and extraneuronal metabolism of 5-hydroxytryptamine in the isolated saphenous vein of the dog
}

Maria Quitéria Paiva, Margarida Caramona, and Walter Osswald

Laboratório de Farmacologia, Faculdade de Medicina, P-4200 Porto, Portugal

Naunyn-Schmiedeberg's Arch Pharmacol (1984) 325:62-68

The formula on page 64 , left column, should read:

$V_{\text {total }}=V_{1}+V_{2}=\frac{V_{\text {max }} \cdot S}{K_{\mathrm{m}}+S}+k \cdot S$ 